\title{
Letter to the Editor: The C-terminal domain of Viral IAP associated factor (cVIAF) is a structural homologue of phosducin: Resonance assignments and secondary structure of the C-terminal domain of VIAF
}

\author{
Jaison Jacob ${ }^{\mathrm{a}, *}$, John M. Louis ${ }^{\mathrm{b}}$, B. W. M. Richter ${ }^{\mathrm{c}, * *}$, Colin S. Duckett ${ }^{\mathrm{c}}$ \& Dennis A. Torchia ${ }^{\mathrm{a}}$ \\ ${ }^{\mathrm{a}}$ Molecular Structural Biology Unit, National Institute of Dental and Craniofacial Research and ${ }^{\mathrm{b}}$ Laboratory of \\ Chemical Physics, National Institute of Diabetes and Digestive and Kidney Diseases, National Institutes of Health, \\ Bethesda, Maryland 20892, U.S.A.; ${ }^{\mathrm{c}}$ Departments of Pathology and Internal Medicine, University of Michigan \\ Medical School, Ann Arbor, Michigan 48109, U.S.A.
}

Received 12 June 2003; Accepted 23 July 2003

Key words: apoptosis, cVIAF, IAP, NMR assignments, phosducin

\section{Biological context}

The iap (inhibitor of apoptosis) gene family (Duckett et al., 1996) are a highly conserved group of factors that are thought to play central roles in the control of apoptosis and cell cycle progression. Several IAPs have been shown to suppress apoptotic cell death through the direct enzymatic suppression of the chief effectors of apoptosis, the caspase family of cysteine proteases (Deveraux and Reed, 1999). VIAF (Viral IAP-Associated Factor) was originally identified as an IAP binding protein through a yeast two-hybrid screen of a human B-cell cDNA library with the prototype IAP (Op-IAP) from the baculovirus Orgyia pseudotsugata multinucleocapsid nuclear polyhedrosis virus (OpMNPV) (B.W.M. Richter et al., manuscript in preparation). Op-IAP is an extremely potent inhibitor of apoptosis even in mammalian cells (Duckett et al., 1996). Preliminary data suggest that VIAF interacts with several mammalian IAPs, most notably survivin and XIAP. We have found that ectopic expression of VIAF alone is modestly cytoprotective, while coexpression of VIAF synergistically enhances the anti-apoptotic properties of either Op-IAP or XIAP. Furthermore, we have found that reduction of endogenous VIAF through RNA interference (RNAi) induces cell cycle arrest.

\footnotetext{
*To whom correspondence should be addressed. E-mail: jaison.jacob@vanderbilt.edu

**Present address: Virion Systems, Inc., Gaithersburg, MD.
}

\section{Methods}

The 152 residue $\mathrm{C}$-terminal fragment of human VIAF was over-expressed in host bacteria BL21-Gold (Stratagene, La Jolla, CA) using the vector pET$16 \mathrm{~b}$ (Novagen, Madison, WI) grown at $37^{\circ} \mathrm{C}$ in a modified minimal medium with ${ }^{15} \mathrm{NH}_{4} \mathrm{Cl}$ as the sole nitrogen source and $\left[{ }^{13} \mathrm{C}_{6}\right]$ glucose as the sole carbon source. The recombinant protein was purified and refolded as described elsewhere (Jacob et al., 2002), and exchanged into a buffer containing $20 \mathrm{mM}$ sodium phosphate $\mathrm{pH} 7.4,0.02 \mathrm{mM}$ EDTA, $0.01 \% \mathrm{NaN}_{3}$ and $2 \mathrm{mM}$ DTT in $95 \% \mathrm{H}_{2} \mathrm{O} / 5 \% \mathrm{D}_{2} \mathrm{O}$ and concentrated to $0.9 \mathrm{mM}$.

NMR measurements were carried out in Shigemi microcells at $35^{\circ} \mathrm{C}$ on Bruker spectrometers: a DMX500, another DMX500 equipped with a cryoprobe, or a DMX 750, all with triple resonance probes and gradients. The backbone assignments were primarily derived from $2 \mathrm{D}-\left[{ }^{15} \mathrm{~N},{ }^{1} \mathrm{H}\right]-\mathrm{HSQC}, 3 \mathrm{D}$ HNCA, 3D HNCACB, 3D CBCA(CO)NH and 3D $\mathrm{HNCO}$ spectra, and sidechain assignments using $3 \mathrm{D}$ CCONH, 3D H(C)CONH and 3D H(C)CH-TOCSY. Stereospecific assignments of isopropyl methyls of Val and Leu were obtained using biosynthetically directed frational ${ }^{13} \mathrm{C}$ labeling. NOEs between $\mathrm{\beta CH}_{2}$ and aromatic protons observed in a $3 \mathrm{D}{ }^{13} \mathrm{C}$-resolved $\left[{ }^{1} \mathrm{H},{ }^{1} \mathrm{H}\right]$-NOESY were used as starting points for sequence specific assignments of aromatic sidechains. These NOEs in combination with $2 \mathrm{D}$ CT- $\left[{ }^{13} \mathrm{C},{ }^{1} \mathrm{H}\right]$ HSQC and homonuclear 2D $\left[{ }^{1} \mathrm{H},{ }^{1} \mathrm{H}\right]$-TOCSY were used to complete the aromatic sidechain assignments. 
Biosynthetically directed fractional ${ }^{13} \mathrm{C}$-labeling and 2D CT- $\left[{ }^{13} \mathrm{C},{ }^{1} \mathrm{H}\right]$-TROSY-HSQC (Jacob et al., 2002) with a $36 \mathrm{~ms}$ CT period afforded the assignments of $\zeta$ carbon and Phe protons. In addition this spectra also helped identify the $\delta$ and $\varepsilon$ position of Phe and Tyr residues. The tautomeric states of the His residues were identified described before (Pelton et al., 1991). All spectra were processed using nmrPipe (Delaglio et al., 1995) and analyzed using PIPP (Garrett et al., 1991).

\section{Extent of assignment and topology}

All non-proline backbone residues with the exception of the amide resonances of residues L126 and $\mathrm{K} 100$ and the preceding $\mathrm{C}^{\prime}$ in the region between K88L220 have been assigned. Several residues between L221-D239 could not be observed due to severe line broadening resulting from intermediate conformational exchange. Over $90 \%$ of the sidechain ${ }^{1} \mathrm{H}$ and ${ }^{13} \mathrm{C}$ resonances of residues in the region K88-L220 have been assigned. The 2 histidine residues are in different tautomeric forms; specifically, $\delta 1$ position of $\mathrm{H} 118$ is protonated while $\mathrm{H} 133$ is in the $\varepsilon 2$ protonation state. Chemical shift index (Wishart and Sykes, 1994) was used to predict secondary structure.

Figure 1 illustrates a close agreement between the predicted secondary structure of VIAF and the crystal structure of the $\mathrm{C}$-terminal domain of phosducin. This observation is not entirely surprising, given the limited sequence homology of VIAF to phosducin like protein $(\mathrm{PhLP})$. As a result of its broad expression pattern in diverse tissues and cell lines, PhLP has been postulated to be a general regulator of $\mathrm{G}$ protein signaling, mediated through its binding to $\mathrm{G}_{\beta \gamma}$ subunit (Thibault et al., 1997). However, more recently PhLP has been found to be involved in other cellular processes via interaction with different binding partners (Zhu and Craft, 1998; Mc Laughlin et al., 2002). While the actual mechanism of VIAF mediated apoptotic regulation is still unidentified, it is clear that VIAF is an important player in the phosducin family of proteins and may provide interesting clues to the general function of other proteins in the phosducin family. The assignments have been deposited in BioMagResBank (http://www.bmrb.wisc.edu) under accession number 5814.

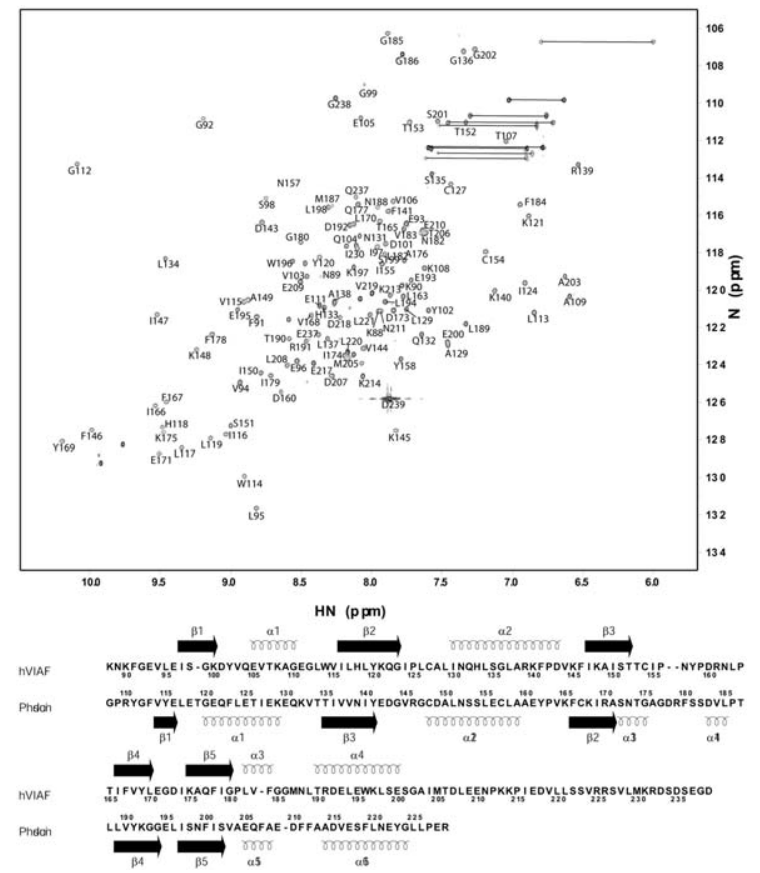

Figure $1 .{ }^{1} \mathrm{H},{ }^{15} \mathrm{~N}-\mathrm{HSQC}$ spectrum of cVIAF at $750 \mathrm{MHz}$ and sequence alignment of the c-terminal domain of VIAF and phosducin. The secondary structures mapped to the sequence show very close structural homology. The secondary structure of VIAF was derived using the CSI method as explained in the text. The secondary structure of phosducin was derived from chain $\mathrm{P}$ in pdb entry 2 TRC.

\section{Acknowledgements}

We thank I. Neshiawat for expert technical assistance, F. Delaglio and D. Garrett for software support and L.K. Pannell for mass spectrometry.

\section{References}

Delaglio, F. et al. (1995) J. Biomol. NMR, 6, 277-293.

Deveraux, Q.L. and Reed, J.C. (1999) Genes Dev., 13, 239-252.

Duckett, C.S. et al. (1996) Embo J., 15, 2685-2694.

Garrett, D.S. et al. (1991) J. Magn. Reson., 95, 214-220.

Jacob, J. et al. (2002) J. Biomol. NMR, 24, 231-235.

McLaughlin, J.N. et al. (2002) Proc. Natl. Acad. Sci. USA, 99, 7962-7967.

Pelton, J.G. et al. (1991) Biochemistry, 30, 10043-10057.

Thibault, C. et al. (1997) J. Biol. Chem., 272, 12253-12256.

Wishart, D.S. and Sykes, B.D. (1994) J. Biomol. NMR, 4, 171-180.

Zhu, X. and Craft, C.M. (1998) Mol. Vis., 4, 13. 